\title{
Simpler Analysis of Serial Transactions Using Reverse Transactions
}

\author{
Karim TRAORE \\ \{karim.traore@ensma.fr\}
}

\author{
Emmanuel GROLLEAU \\ \{grolleau@ensma.fr\}
}

\author{
Francis Cottet \\ \{cottet@ensma.fr\}
}

\author{
LISI/ENSMA \\ Laboratoire d'Informatique Scientifique et Industrielle \\ École Nationale de Mécanique et d'Aérotechnique \\ Téléport 2 - BP 40109 F-86961 Chasseneuil Futuroscope Cedex, France
}

\begin{abstract}
$^{1}$
We present in this article a method of validation for "serial transaction". The serial transaction model has been proposed in order to validate a concrete real-time application. This model is typically a task reading serial information (RS232, CAN,...): several instances are identical and read an unitary part of a serial packet, these tasks have the same WCET, offset shifting, priority and relative deadline. In addition, the last task of a transaction has to deal with the whole packet, and is typically longer, but has a longer relative deadline, and a lower priority. The method of validation that we present here uses the concept of reverse transaction deduced from the serial transaction to analyse.
\end{abstract}

\section{Introduction}

The model of "serial transaction" has been extracted from a real-time application that consists in developing a mini-UAV (Unmanned Air Vehicle) (figure1). In the development of a real-time application like this one, two techniques of scheduling can be used : the on-line scheduling, with a fixed [8][9][1] or variable allocation of priorities of the tasks in the tasks set [2][7][3] and off-line techniques which use a sequence whose correctness was proved [16][5]. One of the most important phases is the temporal validation which consists in proving that whatever happens, all the tasks meet their temporal constraints. RTA (Response Time analysis) methods are used to bound the worst-case response time of the tasks of an application. Tindell [13] proposed a method for calculating an upper bound of the worst-case response time less pessimistic than classic RTA (considering a critical instant consisting of a simultaneous release of all the tasks) in the context of tasks with offsets (transaction).

Palencia and Harbour [12] extended Tindell's work. Lastly, [15][11] introduced the concept of "imposed" interference differing from "released for execution" interference used by Tindell. However, for now the

\footnotetext{
${ }^{1}$ This work was supported by ONERA/DGA
}

exact calculation methods used to determinate the exact worst-case response time relies on calculating every combination of the tasks of the transactions; it thus remains exponential in time.

In order to validate the control system of the UAV, we have to deal with tasks with offset which are particular instances of transactions. First, we present the UAV application in section 2 . Then section 3 presents the concept of transactions and serial transactions. In Section 4, the method of "imposed interference" is defined. In section 5, we present some new results obtained using the concept of reverse transaction.

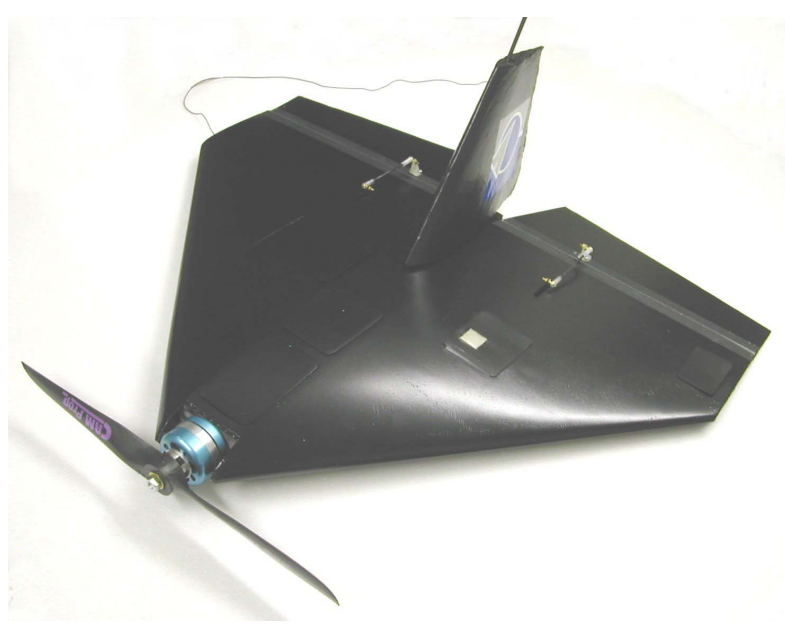

Figure 1: the AMADO

\section{Presentation of the Application}

The project, named AMADO, is a UAV with a wingspread of $55 \mathrm{~cm}$, using a delta shaped wing with two symmetrical drifts for a total weight (including the control system) of 930 grams. The main objective is to create an autonomous plane embedding a camera, and to be able to follow dynamically defined waypoints. The UAV is connected to a ground station thanks to a wireless modem, allowing it to receive high level 
orders during a mission. The critical parts of the flight control are embedded.

\subsection{Description of the application}

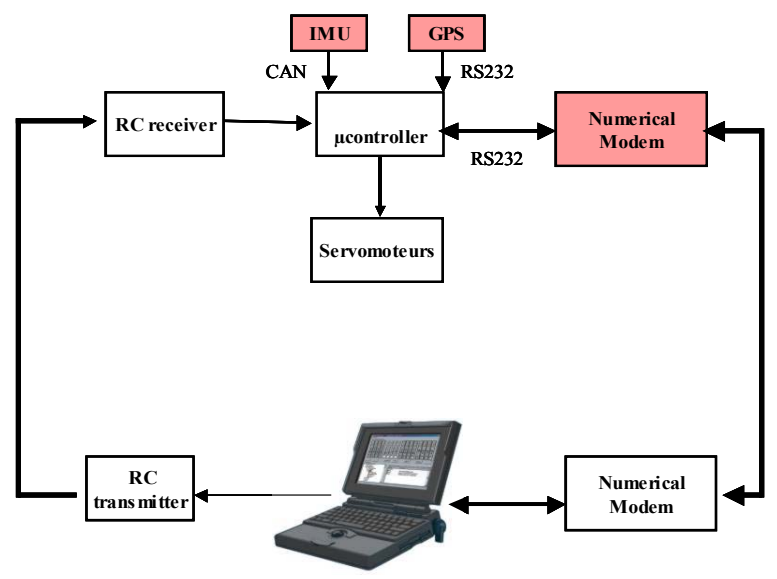

Figure 2: main architecture of the AMADO

The Figure 2 shows two parts: the ground station, and the embedded system.

The embedded system heart is a Freescale/Motorola MPC555[10] connected to the actuators (3 servocommands and the speed-variator, refreshed every 20 $\mathrm{ms}$ ), an IMU [6] (Inertial Measurement Unit), a GPS receiver [4], a traditional radio receiver and a modem. The MPC555 is a 32 bits PowerPC with a frequency of $40 \mathrm{MHZ}, 448 \mathrm{~KB}$ of flash memory and $26 \mathrm{~KB}$ of RAM.

Two sensors are used in order to calculate the position and attitude of the UAV: the GPS receiver and the IMU. The Inertial Measurement Unit sends information about angular speed and accelerations, which, once treated, give the roll and the pitch of the UAV. This IMU is connected on a CAN port and delivers information at a frequency of $50 \mathrm{~Hz}$ and a throughput of $1 \mathrm{Mbps}$. A frame of the IMU is compound of 3 blocks of 6 bytes. In order for the system to get a complete frame, each block must be read before the next arrives. Once the system has 3 blocks, it can constituate the frame, and handle it to calculate the roll and the pitch.

The GPS receiver is used to get the speed (direction and module) and the absolute 3Dimensional position of the UAV. The GPS Receiver sends data to the controller at a frequency of $4 \mathrm{~Hz}$ and delivers information with a throughput of $57600 \mathrm{bps}$. As a RS232 communication, the information is sent byte after byte; the number of bytes sent during one period (frame) of the GPS can reach 120 bytes. As in the case of the IMU, the system must recover each byte and arrange it before the arrival of the next byte, under penalty of losing the complete frame.

Finally the modem [Modem1] connected to the microcontroller on the serial port is bi-directional and communicates with the microcontroller at a throughput of $115 \mathrm{kbps}$. The length of the frame transmitted to the microcontroller by the modem can reach 10 bytes. The requirements are the same as in the case of the GPS receiver. In the presentation of this architecture, we omitted voluntarily the video circuit that does not have any impact on the real-time aspects of this application.

\subsection{Software architecture of the application}

We have chosen the real-time executive OSEKTurbo OS/MPC5xx of Metrowerks[19] for our application. This RTOS is conforming to the standard OSEK/VDX [18]; standard defined for applications with limited resources.

Apart the initialisation task, there are 12 tasks in the control system (see Table 1). The priorities of the tasks have been assigned following a Deadline Monotonic policy [8]. Note that the value $\mathrm{L}=120$ (resp. $\mathrm{L}=3$, $\mathrm{L}=10$ ) corresponds to the number of times the task has to be activated in order to acquire a frame.

\begin{tabular}{|c|c|c|c|c|c|}
\hline Tasks & Period & \multicolumn{2}{|l|}{ WCET } & deadline & Priority \\
\hline & \multicolumn{4}{|c|}{ (in microsecond) } & \\
\hline Monitoring (1) & 200000 & 60 & & 200000 & 1 \\
\hline Acq PWM (2) & 20000 & 24 & & 10000 & 7 \\
\hline Transmit Grd (3) & 50000 & 3360 & & 30000 & 5 \\
\hline Deliver Cmd (4) & 20000 & 40 & & 10000 & 6 \\
\hline Navigation (5) & 250000 & 560 & & 140000 & 2 \\
\hline ReguleAttitude (6) & 60000 & 32400 & & 60000 & 4 \\
\hline Acq GPS (7) & 250000 & 100 & $L=120$ & 160 & 11 \\
\hline Acq IMU (8) & 20000 & 96 & $L=3$ & 720 & 10 \\
\hline Acq Instruction(9) & 100000 & 12 & $L=10$ & 80 & 12 \\
\hline TreatGPS (10) & 250000 & 3000 & & 5000 & 9 \\
\hline TreatIMU (11) & 20000 & 900 & & 7500 & 8 \\
\hline $\begin{array}{l}\text { TreatInstruction } \\
\text { (12) }\end{array}$ & 100000 & 900 & & 70000 & 3 \\
\hline
\end{tabular}

Table1: task system of the UAV

This kind of application cannot be validated easily if the offsets are not taken into account. Indeed, it appears clearly that task TreatGPS is released when the whole GPS frame has been received; it cannot thus be released at the same time as the task Acq GPS; it is the same case for task TreatIMU and the task Acq IMU; the same situation occurs for the task TreatInstruction and the task Acq Instruction.

\section{Presentation of serial transaction}

The Figure 3 presents a model of a serial transaction, $\mathrm{L}_{\mathrm{i}}$ instances of the acquisition of a part of a frame are separated by a duration corresponding to the 
arrival rate of the packets (Acq GPS, Acq IMU, Acq Instruction), and a longer task is used to handle the whole frame (TreatGPS, TreatIMU, TreatInstruction). In a serial transaction, the acquisition tasks are usually short, because they only have to bufferize the packets until the whole frame is built, while the treatment tasks are longer since they have to deal with the full frame.

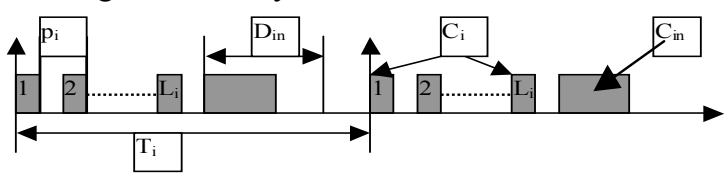

Fig. 3. pattern of a serial transaction

Let us give some results found in [13][14][12] relative to transactions. Certain tasks can have the same period and be bound by relations of offsets i.e. they can never be released at the same time. A set of tasks of the same period bounded by offset is called a transaction. Let us note $\Gamma:=\left\{\Gamma_{1}, \Gamma_{2}, \ldots \Gamma_{\mathrm{k}}\right\}$ a set of transactions. A transaction $\Gamma_{i}$ contains $\left|\Gamma_{\mathrm{i}}\right|$ tasks with a period $T_{i}: \Gamma_{\mathrm{i}}:=$ $<\left\{\tau_{\mathrm{i} 1}, \ldots, \tau_{\mathrm{i}|\Gamma \mathrm{i}|}\right\}, \mathrm{T}_{\mathrm{i}}>\cdot[12][14]$

A task is defined by $\tau_{\mathrm{ij}}:=<\mathrm{C}_{\mathrm{ij}}, \mathrm{O}_{\mathrm{ij}}, \mathrm{D}_{\mathrm{ij}}, \mathrm{J}_{\mathrm{ij}}, \mathrm{B}_{\mathrm{ij}}, \mathrm{P}_{\mathrm{ij}}>$ where $C_{i j}$ is the worst-case execution time (WCET), $\mathrm{O}_{\mathrm{ij}}$ is the offset, $\mathrm{D}_{\mathrm{ij}}$ is the relative deadline, $\mathrm{J}_{\mathrm{ij}}$ the maximum jitter, $B_{i j}$ maximum blocking due to lower priority tasks, and $\mathrm{P}_{\mathrm{ij}}$ the priority. Without loss of generality, we consider that the tasks are ordered by non decreasing offsets $O_{i j}$; in our case, we define the response time as being the time between the release of the task and the completion of the task.

Let us note also $h p_{i}\left(\tau_{u a}\right)$ the set of indices of the tasks of $\Gamma_{i}$ with a priority higher than the priority of a task $\tau_{u a}$ i.e. $\mathrm{j} \in \mathrm{h} \mathrm{p}_{\mathrm{i}}\left(\tau_{\mathrm{ua}}\right)$ if and only if $\mathrm{P}_{\mathrm{ij}}>\mathrm{P}_{\mathrm{ua}}$.

The task under analysis is usually noted $\tau_{u a}$. Tindell showed that the critical instant of $\tau_{u a}$ is a particular instant when it is released at the same time as one task of higher priority in each transaction (its own transaction being handled separately). The main difficulty is to determine what is the critical instant candidate $\tau_{i c}$ of a transaction $\Gamma_{i}$ that initiates the critical instant of $\tau_{u a}$. An exact calculation method would require to evaluate the response time obtained by carrying out all the possible combinations of the tasks of priority higher than $\tau_{u a}$ in each transaction and to choose the task $\tau_{i c}$ in each transaction that leads to the worst-case response time. This exhaustive method has an exponential complexity and is intractable for realistic task systems; several approximation methods exist.

The first method of approximation is the "released for execution" method. This method has been very pessimistic in the case of the application of mini UAV; it has been enable to validate this application however we have proved that the application is valid. Up to now, the best known method of approximation is the "imposed interference" method.

\section{Upper bound method based on the "imposed" interference}

This method has been proposed in [15]; it removes the unnecessary overestimation taken into account in the classic computation of the interference imposed by a task $\tau_{i}$ on a lower priority task $\tau_{u a}$. This overestimation does not have any impact in the case of tasks without offset but has a considerable effect in the approximation of the worst-case response time when we are in the presence of tasks with offsets.. This method consists in calculating the interference effectively imposed by a task $\tau_{j}$ on a task $\tau_{u a}$ with a lower priority during a time interval of length $t$. In order to calculate this "imposed" interference, [15] subtracts a parameter $\mathrm{x}$ (see Figure 2) from the original interference formula; let us note $W_{i c}\left(\tau_{u a}, t\right)$ the interference of $\Gamma_{i}$ on the response time of $\tau_{u a}$ during a time interval of length $\mathrm{t}$ when $\tau_{i c}$ is released at the same instant as $\tau_{u a}$.

$$
\begin{aligned}
& \left.\left.W_{i c}\left(\tau_{u a}, \mathrm{t}\right)=\sum_{j \in h p_{i}\left(\tau_{u a}\right)}\left(\mid t^{*}\right\rfloor+1\right) * C_{i j}-\mathrm{x}_{\mathrm{ijc}}(t)\right) \\
& t^{*}=t-\operatorname{phase}\left(\tau_{\mathrm{ij}}, \tau_{i c}\right) \quad \operatorname{phase}\left(\tau_{\mathrm{ij}}, \tau_{i c}\right)=\left(\mathrm{O}_{\mathrm{ij}}-\mathrm{O}_{\mathrm{ic}}\right) \% \mathrm{~T}_{\mathrm{i}} \\
& x_{i j c}(t)= \begin{cases}0 \quad \text { for } \mathrm{t}^{*}<0 \\
\max \left(0, \mathrm{C}_{\mathrm{ij}}-\left(\mathrm{t}^{*} \% \mathrm{~T}_{\mathrm{i}}\right)\right) & \text { for } \mathrm{t}^{*} \geq 0\end{cases}
\end{aligned}
$$

Example: this transaction has 4 tasks with period $T_{i}=50$

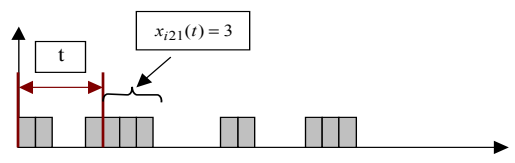

Fig. 2: imposed interference

$$
\begin{aligned}
& \Gamma_{i}:=<\left\{\tau_{i 1}, \tau_{i 2}, \tau_{i 3}, \tau_{i 4}\right\}, 50> \\
& \tau_{i 1}:=<2,0,4,0,0,4> \\
& \tau_{i 2}:=<4,4,8,0,0,2> \\
& \tau_{i 3}:=<2,12,5,0,0,3> \\
& \tau_{i 4}:=<3,17,15,0,0,1> \\
& W_{i 1}\left(\tau_{u a}, 5\right)=(2-0)+(4-3)+(0-0)+(0-0)=3
\end{aligned}
$$

For determining the upper bound of the response-time, we use this function :

$$
W_{i}\left(\tau_{u a}, t\right)=\max _{c \in h p_{i}\left(\tau_{u a}\right)} W_{i c}\left(\tau_{u a}, \mathrm{t}\right)
$$

With the value of each $W_{i}\left(\tau_{u a}, t\right)$, the response time $R_{u a}$ of $\tau_{u a} \quad$ can be calculated. $R_{u a}^{(n+1)}=C_{u a}+\sum_{i \in \Gamma} W_{i}\left(\tau_{u a}, R_{u a}^{n}\right) . R_{u a}$ is obtained by fix- 
point iteration starting with $R_{u a}^{0}=C_{u a}$. Let us execute this method on the example (Figure3 (a))

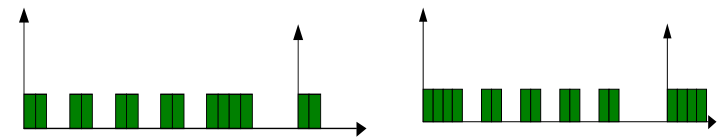

Fig. 3.(a and b) Example for imposed interference (a) reverse transaction (b)

In the transaction $\Gamma_{i}$, we have five tasks. Let us consider a lower priority task $\tau_{u a}$ with $C_{u a}=5$. Let us calculate the response-time. We present at first the details of iteration number 2 :

\section{Iteration 2:}

$W_{i 1}\left(\tau_{u a}, 5\right)=(2-0)+(2-1)+(0-0)+(0-0)+(0-0)=3$

$W_{i 2}\left(\tau_{u a}, 5\right)=(0-0)+(2-0)+(2-1)+(0-0)+(0-0)=3$

$W_{i 3}\left(\tau_{u a}, 5\right)=(0-0)+(0-0)+(2-0)+(2-1)+(0-0)=3$

$W_{i 4}\left(\tau_{u a}, 5\right)=(0-0)+(0-0)+(0-0)+(2-0)+(4-3)=3$

$W_{i 5}\left(\tau_{\text {ua }}, 5\right)=(0-0)+(0-0)+(0-0)+(0-0)+(4-0)=4$

$W_{i}\left(\tau_{u a}, 0\right)=4 \quad R_{u a}=9$

We give the values obtained in the different iterations in the table below:

\begin{tabular}{|l|l|l|l|l|l|l|l|l|}
\hline $\begin{array}{l}\text { Iteration } \\
\text { number }\end{array}$ & $\mathrm{t}$ & $W_{i 1}$ & $W_{i 2}$ & $W_{i} W_{i}$ & $W_{i}$ & $W_{i}$ & $R_{u a}$ \\
\hline 1 & 0 & 0 & 0 & 0 & 0 & 0 & 0 & 5 \\
\hline 2 & 5 & 3 & 3 & 3 & 3 & 4 & 4 & 9 \\
\hline 3 & 9 & 5 & 5 & 5 & 6 & 5 & 6 & 11 \\
\hline 4 & 11 & 6 & 6 & 7 & 6 & 6 & 7 & 12 \\
\hline 5 & 12 & 6 & 6 & 8 & 6 & 6 & 8 & 13 \\
\hline 6 & 13 & 7 & 7 & 8 & 7 & 7 & 8 & 13 \\
\hline
\end{tabular}

Consequently, the value of $R_{u a}$ is equal to 13 .

It is not simple to evaluate the value of imposed interference. Indeed, with this method it is necessary in each iteration to evaluate the value of " $n$ " interferences with " $\mathrm{n}$ " as the number of tasks in the transaction. Moreover, it is necessary to evaluate the value of $" x_{i j c} "$ " $\mathrm{n}$ " times in each iteration. In order to simplify the calculation of the value of imposed interference, we use the transaction presented in figure 3(b). We call this transaction the reverse transaction $\Gamma_{i}^{-1}$ of the transaction $\Gamma_{i}$. With the reverse transaction, we show in the next section that it is sufficient to calculate only the value of $W_{i^{-1} 1}\left(\tau_{u a}, t\right)$ at each iteration.

We present in the table below the values obtained in the different iterations:

\begin{tabular}{|l|l|l|l|l|l|l|}
\hline Iteration Number & 1 & 2 & 3 & 4 & 5 & 6 \\
\hline$W_{i^{-1}} 1$ & 0 & 4 & 6 & 7 & 8 & 8 \\
\hline
\end{tabular}

\section{5- Reverse transaction method}

Definition1: A serial transaction is a transaction with the following constraints:
Let $\Gamma_{i}$ be a serial transaction,

- $\quad$ null jitter: $\forall \mathrm{i} / \tau_{\mathrm{ij}} \in \Gamma_{\mathrm{i}}, \mathrm{J}_{\mathrm{ij}}=0$

- $\quad$ regular arrival pattern $\mathrm{p}_{\mathrm{i}}: \forall \mathrm{j} \in\left[1 . .\left|\Gamma_{\mathrm{i}}\right|\right], \mathrm{O}_{\mathrm{ij}}=(\mathrm{j}-$ 1) $\mathrm{p}_{\mathrm{i}}$

- $\quad$ there are two kinds of tasks :

- the $\mathrm{L}_{\mathrm{i}}=\left|\Gamma_{\mathrm{i}}\right|-1$ acquisition tasks such that : $\tau_{\mathrm{i}, \mathrm{j}, \in[1 . . \mathrm{Li}]}:=<\mathrm{C}_{\mathrm{i}},(\mathrm{j}-1) \mathrm{p}_{\mathrm{i}}, \mathrm{p}_{\mathrm{i}}, 0, \mathrm{~B}_{\mathrm{i},}, \mathrm{P}_{\mathrm{i}}>$;

$\circ$ the treatment task $\tau_{i \Gamma i \mid}:=<C_{\text {in }}, \mathrm{L}_{\mathrm{i}} \mathrm{p}_{\mathrm{i}}, \mathrm{D}_{\text {in }}$, $0, \mathrm{~B}_{\mathrm{ij}}, \mathrm{P}_{\mathrm{in}}>$

with $\quad \mathrm{C}_{\text {in }}>\mathrm{C}_{\mathrm{i}}, \quad \mathrm{D}_{\text {in }}>\mathrm{p}_{\mathrm{i}} \quad, \mathrm{P}_{\text {in }}<\mathrm{P}_{\mathrm{i}} \quad$ and $\left(T_{i}-L_{i} \cdot p_{i}\right)-C_{i n}>p_{i}-C_{i n}$. This means that the treatment task is longer than the acquisition tasks, but is provided a longer deadline and a lower priority.

Definition2 : a task $\tau_{u a}$ is an intermediate priority task for a serial transaction $\Gamma_{i}$ if the priority of $\tau_{u a}$ is lower than acquisition tasks of $\Gamma_{i}$ but higher than the treatment task of $\Gamma_{i}$.

Definition 3 : a task $\tau_{u a}$ is a lower priority task for a serial transaction $\Gamma_{i}$ if the priority of $\tau_{u a}$ is lower than all the tasks of $\Gamma_{i}$.

Definition4: Let $\Gamma_{i}$ be a serial transaction, we call reverse transaction of the serial transaction $\Gamma_{i}$ the transaction $\Gamma_{i}^{-1}$ obtained by putting in first position the task of last position of $\Gamma_{i}$; the other parameters remain identical (period, offsets between tasks, etc.) (see figure 3 (a and b)). The tasks of $\Gamma_{i}^{-1}$ are defined as:

$\begin{array}{ll}\text { - } & \tau_{i^{-1} 1}=<C_{i n}, 0, C_{i n}+\left(p_{i}-C_{i}\right), 0, B_{i\left|\Gamma_{i}\right|}, P_{i\left|\Gamma_{i}\right|}> \\ \text { - } & \tau_{i^{-1} j, j \in\left[2 .\left|\Gamma_{i}\right|\right]}=<C_{i}, C_{i n}-C_{i}+(j-1) \cdot p_{i}, p_{i}, 0, B_{i(j-1)}, P_{i(j-1)}>\end{array}$

Theorem1: Let $\Gamma_{i}$ be a serial transaction, let $\Gamma_{i}^{-1}$ be its reverse transaction and $\tau_{u a}$ a task under analysis. If $\tau_{u a}$ is a lower priority task for the serial transaction $\Gamma_{i}$, then the interference imposed by the serial transaction $\Gamma_{i}$ on the task $\tau_{u a}$ when it is released at the same time as the task initiating the critical instant in $\Gamma_{i}$ has exactly the same value as the interference imposed by $\Gamma_{i}^{-1}$ on $\tau_{u a}$ when $\tau_{u a}$ is released at the same time as the first task in transaction $\Gamma_{i}^{-1}$ i.e $W_{i^{-1} 1}\left(\tau_{u a}, t\right)=W_{i}\left(\tau_{u a}, t\right)$ for any $\mathrm{t}$.

Proof: : Let us note $f_{i}=T_{i}-L_{i} \cdot p_{i}$; and $W_{i^{-1} 1}\left(\tau_{u a}, t\right)$ the imposed interference on the task $\tau_{u a}$ by the transaction $\Gamma_{i}^{-1}$ in a time interval of length $\mathrm{t}$. We thus will calculate $W_{i^{-1} 1}\left(\tau_{u a}, t\right)-W_{i c}\left(\tau_{u a}, t\right)$.

For any time interval of length $\mathrm{t}$, we know that there is an integer k such that $t=k \cdot T_{i}+t \% T_{i}$. 
According

to

$$
\begin{gathered}
W_{i^{-1} 1}\left(\tau_{u a}, t\right)=W_{i^{-1} 1}\left(\tau_{u a}, k \cdot T_{i}\right)+W_{i^{-1} 1}\left(\tau_{u a}, t \% T_{i}\right) \\
W_{i c}\left(\tau_{u a}, t\right)=W_{i c}\left(\tau_{u a}, k \cdot T_{i}\right)+W_{i c}\left(\tau_{u a}, t \% T_{i}\right)
\end{gathered}
$$

Since the value of interference imposed in any time interval of length $T_{i}$ (Period) is the same whatever the beginning of this interval is, then $W_{i^{-1} 1}\left(\tau_{u a}, k \cdot T_{i}\right)=W_{i c}\left(\tau_{u a}, k \cdot T_{i}\right)=k \cdot\left(L_{i} \cdot C_{i}+C_{i n}\right)$ consequently,

$$
\begin{aligned}
& W_{i^{-1} 1}\left(\tau_{u a}, t\right)-W_{i c}\left(\tau_{u a}, t\right)= \\
& W_{i^{-1} 1}\left(\tau_{u a}, t \% T_{i}\right)-W_{i c}\left(\tau_{u a}, t \% T_{i}\right)
\end{aligned}
$$

\begin{tabular}{|c|c|c|}
\hline Interval & $W_{i^{-1} 1}\left(\tau_{u a}, t\right)$ & $W_{i}\left(\tau_{u a}, t\right)$ \\
\hline $\mathrm{t} \leq \mathrm{C}_{\mathrm{in}}$ & $W_{i^{-1} 1}\left(\tau_{u a}, t\right)=t$ & $W_{i n}\left(\tau_{u a}, t\right)=t$ \\
\hline $\begin{array}{l}\mathrm{t} \geq \mathrm{C}_{\mathrm{in}}+ \\
L_{i} \cdot p_{i}\end{array}$ & $\begin{array}{l}W_{i^{-1} 1}\left(\tau_{u a}, t\right)=C_{\text {in }}+ \\
L_{i} \cdot C_{i}\end{array}$ & $\begin{array}{l}W_{i 1}\left(\tau_{u a}, t\right)= \\
\mathrm{C}_{\text {in }}+L_{i} \cdot C_{i}\end{array}$ \\
\hline $\begin{array}{l}C_{\text {in }}<t \\
\text { and } \\
t<C_{i n}+ \\
L_{i} \cdot p_{i}\end{array}$ & $\begin{array}{l}W_{i^{-1} 1}\left(\tau_{u a}, t\right)=C_{i n}+ \\
\left\lceil\frac{t-\left(C_{i n}+\left(p_{i}-C_{i}\right)\right)}{p_{i}}\right\rceil \cdot C_{i} \\
-x_{i^{-1} 1}(t)\end{array}$ & $\begin{array}{l}W_{i}\left(\tau_{u a}, t\right)= \\
\max \left\{\begin{array}{l}\mathrm{W}_{\mathrm{ic}}\left(\tau_{u a}, t\right) \\
\forall \mathrm{c} \in\left[1 . . \mathrm{L}_{\mathrm{i}}+1\right]\end{array}\right.\end{array}$ \\
\hline
\end{tabular}

so we can suppose $0 \leq t<T_{i}$; with this consideration, we have :

For $\mathrm{t} \leq \mathrm{C}_{\mathrm{in}}$ and $\mathrm{C}_{\mathrm{in}}+L_{i} \cdot p_{i} \leq \mathrm{t}<T_{i}$, we have already $W_{i^{-1} 1}\left(\tau_{u a}, t\right)=W_{i}\left(\tau_{u a}, t\right)$

We have now to prove the equality $W_{i^{-1} 1}\left(\tau_{u a}, t\right)=W_{i}\left(\tau_{u a}, t\right)$ for $C_{i n}<t<C_{i n}+L_{i} \cdot p_{i}$.

For $\left.\mathrm{t} \in] \mathrm{C}_{\mathrm{in}} ; \mathrm{C}_{\mathrm{in}}+\left(p_{i}-C_{i}\right)\right], W_{i^{-1} 1}\left(\tau_{u a}, t\right)=W_{i\left(L_{i}+1\right)}\left(\tau_{u a}, t\right)$; c is equal to $L_{i}+1$; and for $\mathrm{t} \in] \mathrm{C}_{\mathrm{in}}+\left(p_{i}-C_{i}\right) ; \mathrm{C}_{\mathrm{in}}+L_{i} \cdot p_{i}\left[, \exists \mathrm{c} \in\left[1 . . L_{i}\right]\right.$ such as $t \in$ ] $\left.\mathrm{C}_{\mathrm{in}}+\left(p_{i}-C_{i}\right)+\left(L_{i}-c\right) \cdot p_{i} ; \mathrm{C}_{\mathrm{in}}+\left(p_{i}-C_{i}\right)+\left(L_{i}-c+1\right) \cdot p_{i}\right]$

For these two cases, we have $W_{i^{-1} 1}\left(\tau_{u a}, t\right)=W_{i c}\left(\tau_{u a}, t\right)$

Moreover, $W_{i c}\left(\tau_{u a}, t\right)=\left(L_{i}-c+1\right) \cdot C_{i}+\left(C_{i n}-x_{i c n}(t)\right)$ because $f_{i}-C_{i n}>p_{i}-C_{i}$ (according to the definition of serial transaction).

We will prove now that for all $p \in\left[1 . . L_{i}+1\right]$, $W_{i p}\left(\tau_{u a}, t\right) \leq W_{i c}\left(\tau_{u a}, t\right)$

We take the value of $W_{i c}\left(\tau_{u a}, t\right)$ like reference (figure 4 (a))

\section{$\underline{1^{\text {st }} \text { case }: p>c \text { with } c<L_{i}+1}$}

It appears clearly on the figure 4 (A and $B$ ) that the shifting of the interval $t$ from $c$ to the position $p$ decreases the value of the interference by $(p-c) \cdot C_{i}$ on the left side whereas the increasing on the value of interference obtained on the right side is lower or equal to $(p-c) \cdot C_{i}$ because $f_{i}-C_{i n}>p_{i}-C_{i}$. Therefore $W_{i p}\left(\tau_{u a}, t\right) \leq W_{i c}\left(\tau_{u a}, t\right)$.

$\underline{2^{\text {nd }} \text { case }: \text { p }<\mathbf{c} \text { with } \mathbf{c}>1}$

On the figure 4 (A and $\mathrm{C}$ ), we can see that every time the interval $\mathrm{t}$ is shifted the value $p_{i}$ towards the left (until we reach the position $\mathrm{p}$ such as $\left.t<\left(L_{i}+1-p\right) \cdot p_{i}\right)$, we add $C_{i}$ on the value of the interference on the left side; however, the decreasing on the value of the interference on the right side is in the interval $\left[C_{i} ; p_{i}\right]$; therefore the value of the interference after this shifting decreases. When $\mathrm{t}$ is lower than $\left(L_{i}+1-p\right) \cdot p_{i}$, a shifting of the interval $\mathrm{t}$ towards the left doesn't change the value of the interference (Figure 4 (D)).

Consequently, the value of $W_{i p}\left(\tau_{u a}, t\right)$ is always lower or equal to the value of $W_{i c}\left(\tau_{u a}, t\right)$.
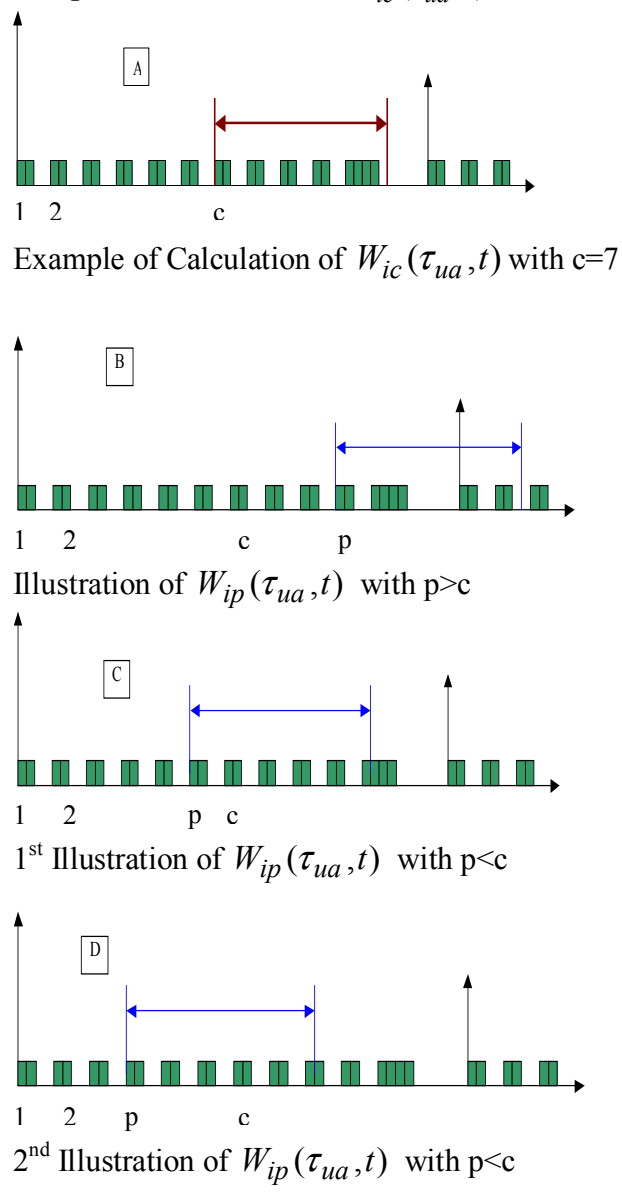

Fig.4 : Illustration of the comparison between $W_{i p}\left(\tau_{u a}, t\right)$ and $W_{i c}\left(\tau_{u a}, t\right)$

Let us note $h p\left(\tau_{u a}\right)$ the set of serial transactions such that $\tau_{u a}$ has a lower priority than every task of the transaction. 
By applying Theorem1, the interference applied by the serial transactions whose indices belong to $h p\left(\tau_{u a}\right)$ in a time interval of length $t$ is :

$$
\begin{aligned}
& \sum_{\mathrm{j} \in \mathrm{hp}\left(\tau_{\text {uа }}\right)}\left(\left\lceil\frac{t}{T_{j}}\right\rfloor \cdot C_{j n}+\left\lfloor\frac{t}{T_{j}}\right\rfloor \cdot L_{j} \cdot C_{j}\right. \\
& \left.+\min \left(\left[\frac{\left(t \% T_{j}-C_{j n}-\left(p_{j}-C_{j}\right)\right)}{p_{j}}\right], L_{j}\right) \cdot C_{j}\right)
\end{aligned}
$$

This formula facilitates the calculation of the upper bound of worst-case response time for the lower priority tasks and allows us to validate the mini UAV application. Indeed, using this formula in conjunction with the formula obtained in [17] for the intermediate priority tasks, we obtained the following values (Table 2) :

\begin{tabular}{|c|c|c|c|c|}
\hline Tasks & Period & deadline & Priority & $\begin{array}{c}\text { Worst-case } \\
\text { response time }\end{array}$ \\
\hline 1 & 200000 & 200000 & 1 & 56156 \\
\hline 2 & 20000 & 10000 & 7 & 6532 \\
\hline 3 & 50000 & 30000 & 5 & 15532 \\
\hline 4 & 20000 & 10000 & 6 & 6572 \\
\hline 5 & 250000 & 140000 & 2 & 56096 \\
\hline 6 & 60000 & 60000 & 4 & 54636 \\
\hline 7 & 250000 & 160 & 11 & 124 \\
\hline 8 & 20000 & 720 & 10 & 468 \\
\hline 9 & 100000 & 80 & 12 & 12 \\
\hline 10 & 250000 & 5000 & 9 & 3408 \\
\hline 11 & 20000 & 7500 & 8 & 5620 \\
\hline 12 & 100000 & 70000 & 3 & 55416 \\
\hline
\end{tabular}

Table2: Calculation of tighter upper bound of worst-case response time of all the tasks of the UAV application

In the table 2, we can see that all the upper bound worst-case response times are lower than the deadline; consequently the application of the UAV is valid.

\section{6- Conclusion}

In this article, we have at first presented the model of serial transactions. A serial transaction $\Gamma_{\mathrm{i}}$ is compound with $\mathrm{L}_{\mathrm{i}}$ short but urgent acquisition tasks activated each time a serial packet is received, and a less urgent but longer treatment task activated when a whole frame is received.

The number of acquisition tasks can be important and makes the exact calculation of response time intractable. Then, we have presented the imposed interference method that gives a tighter upper bound .

After these presentations, we have introduced the concept of reverse transaction that simplify the way to evaluate the imposed interference of a transaction. Our future work will focus on the method to determine the real worst case response-time in the context of serial transactions.

\section{References}

1. N.C. Audsley, Optimal priority assignment and feasibility of static priority tasks with arbitrary start times, Tech. Report YCS-164, University of York, nov. 1991.
2. M.L. Dertouzos, Control robotics : the procedural control of physical processors,

Proc. of IFIP Congress, 1974, pp. 807-813.

3. M.L. Dertouzos, A.K. Mok, Multiprocessor on-line scheduling of hard real-time tasks, IEEETransactions on Software Engineering 15(12), Déc. 1989, 1497-1506.

4. TIM-LC, TIM-LF, TIM-LP System Integration Manual, http://www.u-blox.com

5. E. Grolleau, Ordonnancement temps réel hors-ligne optimal à l'aide de réseaux de pétri en environnement monoprocesseur et multiprocesseur, thèse, ENSMA Université de Poitiers, nov. 1999.

6. Crista Inertial Measurement Unit (IMU) Interface / Operation Document, May 2004, http://www.cloudcaptech.com.

7. J. Labetoulle, Un algorithme optimal pour la gestion des processus en temps réel, Revue Française d'Automatique, Informatique et Recherche Opérationnelle (Fév.1974), 1117.

8. C.L. Liu and J.W. Layland, Scheduling algorithms for mutltiprogramming in real-time environnement, Journal of the ACM 20(1) (1973), 46-61.

9. J. Leung and J. Whitehead, On the complexity of fixedpriority scheduling of periodic, real-time tasks, Performance Evaluation (Netherland) 2(4) (1982), p.237250.

10.MPC555/MPC556 User's Manual October 2000, http://ewww.motorola.com

11.J. Mäki-Turja and M. Sjödin, Improved Analysis for RealTime Tasks With Offsets - Advanced Model. Technical Report MRTC no. 101, Mälardalen Real-Time Research Centre(MRTC), May 2003.

12.J.Palencia Gutierrez and M.Gonzalez Harbour. Schedulability Analysis for Tasks with Static and Dynamic Offsets. In Proc. $19^{\text {th }}$ IEEE Real-Time System Symposium (RTSS), December 1998

13.K. Tindell, Addind Time-Offsets to Schedulability Analysis, Technical Report YCS 221, Dept of Computer Science, University of York, England, January 1994

14.J.Mäki-Turja and M.Nolin. Faster Response Time Analysis of Tasks with Offsets. In Proc. 10 $0^{\text {th }}$ IEEE RealTime Technology and Applications Symposium (RTAS), May 2004

15.J.Mäki-Turja and M.Nolin. Tighter Response Time Analysis of Tasks with Offsets. In Proc. $10^{\text {th }}$ International conference on Real-Time Computing and Applications (RTCSA'04), August 2004

16.J. $\mathrm{Xu}$ and D.L. Parnas, Pre-run-time scheduling of processes with exclusion relations on nested or overlapping critical sections, Phoenix Conference on Computers and Communications (Phoenix, USA), Apr. 1992,pp. 6471-6479.

17. K.Traore, E.Grolleau and F.Cottet, Efficient Schedulability Analysis of Serial Transactions, rapport de recherche $\mathrm{nr}$ 06001, Laboratoire d'Informatique Scientifique et Industrielle ENSMA, Janvier 2006

18.OSEK/VDX operating system specification 2.2.2 July 2002, http://www.osekvdx.org.

19.OSEKturbo OS/MPC5xx User's Manual, Juin 2003, http://www.metrowerks.com. 